\title{
RECONSTRUCTION OF ENERGY-DEPENDENT STURM-LIOUVILLE EQUATIONS FROM TWO SPECTRA
}

\author{
NATALIYA PRONSKA
}

\begin{abstract}
In this paper we study the inverse spectral problem of reconstructing energy-dependent Sturm-Liouville equations from two spectra. We give a reconstruction algorithm and establish existence and uniqueness of reconstruction. Our approach essentially exploits the connection between the spectral problems under study and those for Dirac operators of a special form.
\end{abstract}

\section{INTRODUCTION}

In this paper, we consider energy-dependent Sturm-Liouville differential equations

$$
-y^{\prime \prime}+q y+2 \lambda p y=\lambda^{2} y
$$

on $(0,1)$; here $\lambda \in \mathbb{C}$ is the spectral parameter, $p$ is a real-valued function from $L_{2}(0,1)$ and $q$ is a real-valued distribution from the Sobolev space $W_{2}^{-1}(0,1)$, i.e. $q=r^{\prime}$ with a real-valued $r \in L_{2}(0,1)$ (see a detailed definition in the next section). Our aim is to study the inverse problem of reconstructing the potential $p$ and a primitive $r$ of $q$ from the spectra of the problem (1.1) under two types of boundary conditions: the Dirichlet ones

$$
y(0)=y(1)=0
$$

and the so-called mixed conditions

$$
y(0)=\left(y^{\prime}-r y\right)(1)=0 .
$$

The spectral problems under consideration often arise in classical and quantum mechanics. The most common example is modelling of the motion of massless particles such as photons by means of the Klein-Gordon equations, which can be transformed to Sturm-Liouville

Date: November 23, 2018.

2010 Mathematics Subject Classification. Primary 34A55, Secondary 34B07, 34B24, 34B30, 34L40, 47E05.

Key words and phrases. Inverse spectral problem, energy-dependent potentials, Sturm-Liouville operators. 
equations with potentials depending on the spectral parameter. The corresponding evolution equations are used to describe interactions of colliding spinless particles. The equations of interest also arise in modelling of mechanical systems vibrations in viscous media, see [46]. As the spectral parameter is related to the energy of the system, this explains the terminology "energy-dependent potentials" widely accepted in the physical and mathematical literature.

Since equation (1.1) depends nonlinearly on $\lambda$, we should regard the spectral problems (1.1), (1.2) and (1.1), (1.3) as those for quadratic operator pencils. Some spectral properties of such problems can be derived from the general spectral theory of polynomial operator pencils 31 .

An interesting approach to the spectral analysis of Klein-Gordon equations, using the theory of Krein spaces (i.e. spaces with indefinite scalar products) was suggested by P. Jonas [22] and H. Langer, B. Najman, and C. Tretter [26, 27, 36]. This method was also applied to the spectral analysis of problems of interest, cf. [38].

Equation (1.1) has appeared earlier in the context of inverse scattering. For example, M. Jaulent and C. Jean studied inverse scattering problems for energy-dependent Schrödinger operators on the line and half-line in [18 21]. Such problems were also considered in $[1,23,29,30,32,33,39,44,45]$.

However, the inverse problems for energy-dependent Sturm-Liouville equations on a finite interval have not been studied sufficiently well. M. Gasymov and G. Guseinov discussed such problems with $p \in$ $W_{2}^{1}(0,1)$ and $q \in L_{2}(0,1)$ and with Robin boundary conditions in their short paper [8] of 1981 containing no proofs. In the papers [9, 10, 34, 35, 47] the spectral problems of interest with (quasi)-periodic boundary conditions were considered, but only the Borg-type uniqueness results were obtained therein.

In the present paper, we consider the inverse spectral problem for (1.1) under minimal smoothness assumptions on the real-valued potentials $p$ and $q$, including, e.g. the case when $q$ contains Dirac deltafunctions and/or Coulumb-like singularities. Such functions often arise in modelling of interactions in molecules and atoms in quantum mechanics, see the monographs by S. Albeverio et al. [2] and by S. Albeverio and P. Kurasov [4] and the extensive reference lists therein. The inverse spectral problem for equation (1.1) under such minimal smoothness assumptions on the potentials $p$ and $q$ was considered in [14], where the reconstruction from the Dirichlet spectrum and the set of suitably defined norming constants was investigated. 
Here, we study the problem of reconstruction of the potentials $p$ and $q$ of (1.1) from the two spectra determined respectively by the boundary conditions (1.2) and (1.3). Namely, we give a complete description of these spectra; establish constructively existence of the pencil with given spectra and thus derive a reconstructing algorithm; finally, we prove uniqueness of the reconstruction. Note that (1.3) contains an unknown primitive $r$ of $q$; therefore it is in fact this primitive $r$ (called the regularized potential, or simply the potential) rather than just $q$ that should be found. Our approach uses the technique analogous to that of [14. Namely, we shall associate the spectral problems (1.1), (1.2) and (1.1), (1.3) with those for Dirac operators of a special form and shall essentially use the well-developed inverse spectral theory for Dirac operators in our analysis.

The paper is organized as follows. In the next section we give the explicit formulation of the inverse problem of interest and state the main results. In Section 3, we show that the spectral problems under consideration can be reduced to those for Dirac operators with potentials of a special form. In Section 4, we introduce the so-called transformation operators relating such Dirac operators to the Dirac operators in a canonical form. Existence and uniqueness of Dirac operators with potential of the special form having given sets as their spectra is established in Section 5: in this section we also prove the main results. In the last section we formulate the reconstruction algorithm and discuss some possible extensions.

Notations. Throughout the paper, we denote by $L_{2, \mathbb{R}}(0,1)$ and $W_{2, \mathbb{R}}^{-1}(0,1)$ the spaces of real-valued functions in $L_{2}(0,1)$ and distributions in $W_{2}^{-1}(0,1)$, respectively, and by $\mathcal{M}_{2}=\mathcal{M}_{2}(\mathbb{C})$ the linear space of $2 \times 2$ matrices with complex entries endowed with the Euclidean operator norm. Next, $\rho(T)$ and $\sigma(T)$ will stand for the resolvent set and the spectrum of a linear operator or a quadratic operator pencil $T$. The superscript t will signify the transposition of vectors and matrices, e.g. $\left(c_{1}, c_{2}\right)^{\mathrm{t}}$ is the column vector $\left(\begin{array}{l}c_{1} \\ c_{2}\end{array}\right)$.

\section{Preliminaries AND MAin Results}

As was mentioned before, the spectral problems (1.1), (1.2) and (1.1), (1.3) are the spectral problems for quadratic operator pencils. To give a precise definition, we consider first the differential expression

$$
\ell(y):=\frac{-y^{\prime \prime}}{3}+q y .
$$


Recall that $q=r^{\prime}$ is a real-valued distribution in the Sobolev space $W_{2}^{-1}(0,1)$, and to define $\ell$ rigorously one can use, e.g. the regularization by quasiderivative method due to Savchuk and Shkalikov [40,41]. Following that method, one introduces the quasi-derivative $y^{[1]}:=y^{\prime}-r y$ of an absolutely continuous $y$ and then define $\ell$ as

$$
\ell(y)=-\left(y^{[1]}\right)^{\prime}-r y^{[1]}-r^{2} y
$$

on the set

$$
\operatorname{dom} \ell=\left\{y \in A C(0,1) \mid y^{[1]} \in A C[0,1], \ell(y) \in L_{2}(0,1)\right\} .
$$

It is easy to verify that $\ell(y)=-y^{\prime \prime}+q y$ in the sense of distributions and that for regular potentials $q$ the above definition coincides with the standard one.

Denote by $A_{1}$ and $A_{2}$ Sturm-Liouville operators defined via

$$
A_{j} y=\ell(y), \quad j=1,2,
$$

on the domains

$$
\operatorname{dom} A_{1}:=\left\{y \in \operatorname{dom} \ell \mid y(0)=y^{[1]}(1)=0\right\}
$$

and

$$
\operatorname{dom} A_{2}:=\{y \in \operatorname{dom} \ell \mid y(0)=y(1)=0\} .
$$

For $q \in W_{2, \mathbb{R}}^{-1}(0,1)$ the operators $A_{1}$ and $A_{2}$ are known [40,41] to be self-adjoint, bounded below, and to have simple discrete spectra.

Further, we denote by $B$ the operator of multiplication by the potential $p \in L_{2}(0,1)$. The operator $B$ is in general unbounded. However, since the Green functions of the operators $A_{j}$ are continuous on the square $[0,1] \times[0,1]$, the resolvents of $A_{j}$ are of Hilbert-Schmidt class. It follows that for every non-real $\lambda$ the operators $B(A-\lambda)^{-1}$, $j=1,2$, are also integral operators of Hilbert-Schmidt class; in par-

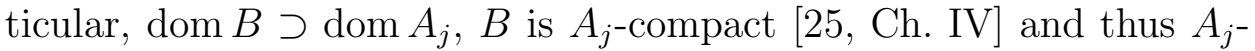
bounded with relative $A_{j}$-bound 0 [6, Lemma III.2.16]. As usual, $I$ shall stand for the identity operator in $L_{2}(0,1)$.

Now the spectral problems (1.1), (1.2) and (1.1), (1.3) can be regarded as the spectral problems for the quadratic operator pencils $T_{2}(p, r)$ and $T_{1}(p, r)$ respectively, given by

$$
T_{j}(p, r)(\lambda):=\lambda^{2} I-2 \lambda B-A_{j}, \quad j=1,2,
$$

for $\lambda \in \mathbb{C}$ on the $\lambda$-independent domain $\operatorname{dom} T_{j}(p, r):=\operatorname{dom} A_{j}$. From the above-listed properties of the operators $A_{j}$ and $B$ we conclude that for every $\lambda \in \mathbb{C}$, the operator $T_{j}(p, r)(\lambda)$ is well defined and closed on $\operatorname{dom} T_{j}(p, r)$.

We now recall the following notions of the spectral theory of operator pencils, see [31]. 
Definition 2.1. An operator pencil $T$ is an operator-valued function on $\mathbb{C}$. The spectrum $\sigma(T)$ of an operator pencil $T$ is the set of all $\lambda \in \mathbb{C}$ for which $T(\lambda)$ is not boundedly invertible, i.e.

$$
\sigma(T)=\{\lambda \in \mathbb{C} \mid 0 \in \sigma(T(\lambda))\} .
$$

A number $\lambda \in \mathbb{C}$ is called the eigenvalue of $T$ if $T(\lambda) y=0$ for some non-zero function $y \in \operatorname{dom} T$, which is then the corresponding eigenfunction. Finally,

$$
\rho(T):=\mathbb{C} \backslash \sigma(T)
$$

is the resolvent set of an operator pencil $T$.

It was shown in [38] that the spectra of the operator pencils $T_{j}(p, r)$, $j=1,2$, consist entirely of eigenvalues and that $\sigma\left(T_{j}(p, r)\right)$ are discrete subsets of $\mathbb{C}$. There are examples of $T_{j}(p, r)$ having non-real and/or non-simple eigenvalues; the latter means that the algebraic multiplicity of an eigenvalue can be greater than 1 [31]. In such a generic setting, the inverse spectral problem of reconstructing the potentials $p$ and $q$ in (1.1) is very complicated, and only partial existence results have been established so far. The approach we are going to use gives a complete solution in the special case where the spectra of $T_{1}(p, r)$ and $T_{2}(p, r)$ are real and simple. A sufficient condition for this to hold is, e.g., that there is a $\mu_{*} \in \mathbb{R}$ such that the operator $T_{1}(p, r)\left(\mu_{*}\right)$ is negative [38]. Thus our standing assumption is that

(A) the operator pencil $T_{1}(p, r)$ is hyperbolic [31, Ch. IV, §31], or, equivalently, there is a $\mu_{*} \in \mathbb{R}$ such that the operator $T_{1}(p, r)\left(\mu_{*}\right)$ is negative.

Under assumption (A), the eigenvalues $\mu_{n}, n \in \mathbb{Z}$, of $T_{1}(p, r)$ and $\lambda_{n}$, $n \in \mathbb{Z}^{*}:=\mathbb{Z} \backslash\{0\}$, of $T_{2}(p, r)$ are all real, simple (see [38]) and can be labelled in increasing order so that $\mu_{n}$ and $\lambda_{n}$ obey the asymptotics

$$
\mu_{n}=\pi\left(n-\frac{1}{2}\right)+p_{0}+\tilde{\mu}_{n}, \quad \lambda_{n}=\pi n+p_{0}+\tilde{\lambda}_{n},
$$

with $p_{0}:=\int_{0}^{1} p(x) d x$ and $\left(\tilde{\mu}_{n}\right),\left(\tilde{\lambda}_{n}\right)$ in $\ell_{2}$ (see [37]) and almost interlace in the sense that $\mu_{k}<\lambda_{k}<\mu_{k+1}$ when $k \in \mathbb{Z}^{*}$ (see [13] and Section [3). Thus the pair of spectra $\left(\left(\lambda_{n}\right),\left(\mu_{n}\right)\right)$ forms then an element of the set $S D$ defined as follows.

Definition 2.2. We denote by $S D$ the family of all pairs $(\boldsymbol{\lambda}, \boldsymbol{\mu})$ of increasing sequences $\boldsymbol{\lambda}:=\left(\lambda_{n}\right)_{n \in \mathbb{Z}^{*}}$ and $\boldsymbol{\mu}:=\left(\mu_{n}\right)_{n \in \mathbb{Z}}$ of real numbers, which satisfy the following conditions:

(i) asymptotics: there is an $h \in \mathbb{R}$ such that

$$
\lambda_{n}=\pi n+h+\tilde{\lambda}_{n}, \quad \mu_{n}=\pi\left(n-\frac{1}{2}\right)+h+\tilde{\mu}_{n},
$$

where $\left(\tilde{\lambda}_{n}\right)$ is a sequence in $\ell_{2}\left(\mathbb{Z}^{*}\right)$ and $\left(\tilde{\mu}_{n}\right)$ is from $\ell_{2}(\mathbb{Z})$; 
(ii) almost interlacing:

$$
\mu_{k}<\lambda_{k}<\mu_{k+1} \quad \text { for every } k \in \mathbb{Z}^{*} \text {. }
$$

Take an arbitrary $\lambda_{0} \in\left(\mu_{0}, \mu_{1}\right)$ and denote by $\boldsymbol{\lambda}^{*}$ the sequence $\boldsymbol{\lambda}$ augmented with $\lambda_{0}$. Then the almost interlacing condition (2.2) means that the sequences $\boldsymbol{\lambda}^{*}$ and $\boldsymbol{\mu}$ strictly interlace.

Remark 2.3. Assume (A) and let the enumeration of the eigenvalues of $T_{1}(p, r)$ and $T_{2}(p, r)$ agree with (2.2); then the number $\mu_{*}$ in assumption (A) belongs to $\left(\mu_{0}, \mu_{1}\right)$. Moreover, the operator $T_{1}(p, r)(\lambda)$ is negative for every $\lambda \in\left(\mu_{0}, \mu_{1}\right)$, so that $(\mathrm{A})$ holds with every $\mu_{*} \in\left(\mu_{0}, \mu_{1}\right)$. The same holds for $T_{2}(p, r)(\mu)$; in particular, then $T_{2}(p, r)(\lambda)$ is negative for every $\lambda \in\left(\mu_{0}, \mu_{1}\right)$.

Denote by $\boldsymbol{\mu}$ and $\boldsymbol{\lambda}$ the spectra of the operator pencils $T_{1}(p, r)$ and $T_{2}(p, r)$ respectively. Then the inverse spectral problem of interest is to reconstruct the operator pencils $T_{j}(p, r), j=1,2$, given the spectra $\boldsymbol{\mu}$ and $\boldsymbol{\lambda}$. Namely, we want to reconstruct the potential $p$ and the (regularized) potential $r$ (determining both the potential $q=r^{\prime}$ and the right-end boundary condition for the operator pencil $T_{1}(p, r)$ in (1.3) ). Some properties of the sets $\boldsymbol{\mu}$ and $\boldsymbol{\lambda}$ are given in [38; our aim here is to give a complete characterization of these spectral data and to find an algorithm reconstructing the potentials $p$ and $r$ from the spectral data.

It is easy to see that with $p \equiv 0$, the spectral problems for the operator pencils $T_{j}(0, r)$ become the usual spectral problems $A_{j} y=\lambda^{2} y$ for the Sturm-Liouville operators $A_{j}$ with potential $q$ in $W_{2}^{-1}(0,1)$; see [40,41]. In this case parts of the sequences $\boldsymbol{\mu}$ and $\boldsymbol{\lambda}$ are redundant in the sense that $\mu_{1-n}=-\mu_{n}$ and $\lambda_{-n}=-\lambda_{n}$. In [16] the authors proved that, just as in the regular case when $q$ is integrable, the spectra $\left(\mu_{n}^{2}\right)_{n \in \mathbb{N}}$ of $A_{1}$ and $\left(\lambda_{n}^{2}\right)_{n \in \mathbb{N}}$ of $A_{2}$ uniquely determine the regularized potential $r$; see also [42] for an alternative treatment. These papers also suggest the algorithm of reconstructing the potential $r$ from the spectra of $A_{1}$ and $A_{2}$.

In the inverse spectral problem of interest we want to determine two real-valued potentials $p$ and $r$ of the operator pencils $T_{j}(p, r), j=1,2$. Since the information contained in the spectra of $T_{j}(p, r)$ is twice as large as for the standard Sturm-Liouville operators one may hope that the inverse spectral problem of reconstructing $p$ and $r$ from the spectral data of $T_{j}(p, r)$ is well posed.

As was already mentioned, the spectra of the operator pencils $T_{j}(p, r)$, $j=1,2$, form an element of $S D$. Our main result states that, conversely, every element of the set $S D$ coincides with the spectral data 
for some operator pencils $T_{j}(p, r)$ under consideration and that these operator pencils are uniquely determined by their spectra.

Theorem 2.4. Assume that a pair $(\boldsymbol{\lambda}, \boldsymbol{\mu})$ of sequences of real numbers is an element of $S D$. Then there exist unique $p, r \in L_{2, \mathbb{R}}(0,1)$ such that $\boldsymbol{\mu}$ and $\boldsymbol{\lambda}$ are the spectra of the pencils $T_{1}(p, r)$ and $T_{2}(p, r)$ respectively. Moreover, the operator pencil $T_{1}(p, r)$ satisfies assumption (A).

The proof of this theorem is constructive and suggests the reconstruction algorithm determining the potentials $p$ and $r$ from the sets $\boldsymbol{\lambda}$ and $\boldsymbol{\mu}$, see Section 6 .

As was already mentioned, our approach consists in reducing the spectral problems for operator pencils $T_{j}(p, r), j=1,2$, to the spectral problems for Dirac operators in $L_{2}(0,1) \times L_{2}(0,1)$ with appropriate boundary conditions, see Section 3. Using a suitable unitary gauge transformation we can reduce these Dirac operators to those in the "shifted" AKNS normal form. For AKNS Dirac operators, the direct and inverse spectral problems are well understood, see [3, 7, 28]. Using known methods we shall reconstruct the AKNS Dirac operators from the given spectra and then transform them to the Dirac operators directly associated with the operator pencils of interest keeping the spectra unchanged. This will give the sought potentials $p$ and $r$ in an explicit form, see (5.6).

\section{Reduction to the Dirac System}

In this section we are going to show that under assumption (A) the spectral problems for the operator pencils $T_{1}(p, r)$ and $T_{2}(p, r)$ can be reduced to the spectral problems for special Dirac operators. The reduction crucially relies on the fact that the equation $y^{\prime \prime}=q_{*} y$ with $q_{*}:=q+2 \mu_{*} p-\mu_{*}^{2}$ and $\mu_{*}$ from (A) has a solution which is strictly positive on $[0,1]$.

Observe first that this equation can be recast as $\ell(y)=\mu_{*}^{2} y-2 \mu_{*} p y$ and treated as the first order linear system $u_{1}^{\prime}=r u_{1}+u_{2}, u_{2}^{\prime}=$ $\left(2 \mu_{*} p-\mu_{*}^{2}-r^{2}\right) u_{1}-r u_{2}$, where $u_{1}=y$ and $u_{2}=y^{[1]}$. Therefore for every complex $a$ and $b$ it possesses a unique solution satisfying the conditions $y(1)=a$ and $y^{[1]}(1)=b$. Let $z$ denote the solution of the equation $y^{\prime \prime}=q_{*} y$ subject to the conditions

$$
z(1)=1, \quad z^{[1]}(1)=0 .
$$

We are going to show that $z$ does not vanish on $[0,1]$.

Lemma 3.1. Under the standing assumption $(A)$ the function $z$ is strictly positive on $[0,1]$. 
Proof. By assumption, the operator $-T_{1}(p, r)\left(\mu_{*}\right)$ is uniformly positive and thus such is the closure $\mathfrak{t}_{1}$ of its quadratic form. For $y \in$ $\operatorname{dom} T_{1}(p, r)$ we find by integration by parts that

$\mathfrak{t}_{1}[y]=\left(-T_{1}(p, r)\left(\mu_{*}\right) y, y\right)=\int_{0}^{1}\left|y^{\prime}\right|^{2}+\int_{0}^{1}\left(2 \mu_{*} p-\mu_{*}^{2}\right)|y|^{2}-2 \operatorname{Re}\left(r y^{\prime}, y\right)$.

The quadratic form $\mathfrak{t}_{0}[y]=\int_{0}^{1}\left|y^{\prime}\right|^{2}$ considered on $\operatorname{dom} T_{1}(p, r)$ is closable and its closure $\tilde{\mathfrak{t}}_{0}$ acts by the same formula on the domain

$$
\operatorname{dom} \tilde{\mathfrak{t}}_{0}:=\left\{u \in W_{2}^{1}(0,1) \mid u(0)=0\right\} .
$$

Since the quadratic form $\int_{0}^{1}\left(2 \mu_{*} p-\mu_{*}^{2}\right)|y|^{2}-2 \operatorname{Re}\left(r y^{\prime}, y\right)$ is relatively bounded with respect to the form $\tilde{\mathfrak{t}}_{0}[y]$ with relative bound 0 (cf. [15]), Theorem VI.1.33 of [25] implies that the domain of $\mathfrak{t}_{1}$ coincides with $\operatorname{dom} \tilde{\mathfrak{t}}_{0}$, i.e.,

$$
\operatorname{dom} \mathfrak{t}_{1}=\left\{u \in W_{2}^{1}(0,1) \mid u(0)=0\right\} .
$$

Now assume that $z$ has zeros on $[0,1]$ and denote the largest of them by $x_{0}$. Then the function

$$
u(x):= \begin{cases}0, & x<x_{0} \\ z(x), & x \geq x_{0}\end{cases}
$$

belongs to the domain of $\mathfrak{t}_{1}$ and is not identically zero. It is straightforward that $\mathfrak{t}_{1}[u]=0$, which contradicts positivity of $-T_{1}(p, r)\left(\mu_{*}\right)$. Therefore $z$ does not vanish on $[0,1]$ yielding the assertion of the lemma.

Setting $v:=z^{\prime} / z$, one easily verifies that $q_{*}=v^{\prime}+v^{2}$; hence $q_{*}$ is a Miura potential [24] and the differential expression $-u^{\prime \prime}+q_{*} u$ can be written in the factorized form, viz.

$$
-u^{\prime \prime}+q_{*} u=-\left(\frac{d}{d x}+v\right)\left(\frac{d}{d x}-v\right) u .
$$

Since $z$ satisfies $\left(z^{\prime}-r z\right)(1)=0$, we have that $(v-r)(1)=0$.

For $\lambda \neq \mu_{*}$ consider the functions $u_{2}:=y$ and $u_{1}:=\left(y^{\prime}-v y\right) /\left(\lambda-\mu_{*}\right)$. Then equation (1.1) can be recast as the following first order system for $u_{1}$ and $u_{2}$,

$$
\begin{aligned}
u_{2}^{\prime}-v u_{2} & =\left(\lambda-\mu_{*}\right) u_{1}, \\
-u_{1}^{\prime}-v u_{1}+2 p u_{2} & =\left(\lambda+\mu_{*}\right) u_{2} .
\end{aligned}
$$

Setting

$$
J:=\left(\begin{array}{cc}
0 & 1 \\
-1 & 0
\end{array}\right), \quad P:=\left(\begin{array}{cc}
\mu_{*} & -v \\
-v & 2 p-\mu_{*} \\
8 &
\end{array}\right), \quad \mathbf{u}(x)=\left(\begin{array}{l}
u_{1} \\
u_{2}
\end{array}\right)
$$


we see that the above system is the spectral problem $\ell(P) \mathbf{u}=\lambda \mathbf{u}$ for a Dirac differential expression $\ell(P)$ acting in $L_{2}(0,1) \times L_{2}(0,1)$ via

$$
\ell(P) \mathbf{u}=J \frac{d \mathbf{u}}{d x}+P \mathbf{u}
$$

on suitable domain.

Denote by $\mathscr{D}_{j}(P), j=1,2$, the Dirac operators generated by $\ell(P)$ on the domains

$\operatorname{dom} \mathscr{D}_{j}(P):=\left\{\mathbf{u}=\left(u_{1}, u_{2}\right)^{\mathrm{t}} \in W_{2}^{1}(0,1) \times W_{2}^{1}(0,1) \mid u_{2}(0)=u_{j}(1)=0\right\}$.

It turns out that the spectra of $\mathscr{D}_{j}(P)$ and $T_{j}(p, r)$ are closely related.

Lemma 3.2. The spectra of the Dirac operators $\mathscr{D}_{j}(P)$ and the operator pencils $T_{j}(p, r), j=1,2$, are related in the following way:

$$
\begin{aligned}
\sigma\left(\mathscr{D}_{1}(P)\right) & =\sigma\left(T_{1}(p, r)\right), \\
\sigma\left(\mathscr{D}_{2}(P)\right) & =\sigma\left(T_{2}(p, r)\right) \cup\left\{\mu_{*}\right\} .
\end{aligned}
$$

Moreover, $y$ is an eigenfunction of $T_{j}(p, r)$ corresponding to an eigenvalue $\lambda$ if and only if $\mathbf{u}=\left(u_{1}, u_{2}\right)^{\mathrm{t}}$ with $u_{1}:=\left(y^{\prime}-v y\right) /\left(\lambda-\mu_{*}\right)$ and $u_{2}=y$ is an eigenfunction of $\mathscr{D}_{j}(P)$ corresponding to $\lambda \neq \mu_{*}$.

Proof. It is straightforward to see that $\lambda=\mu_{*}$ is an eigenvalue of the Dirac operator $\mathscr{D}_{2}(P)$, the corresponding eigenfunction being $\mathbf{u}=\left(u_{1}, u_{2}\right)^{\mathrm{t}}$ with $u_{1}=\exp \left(-\int v\right)$ and $u_{2} \equiv 0$. However, by Remark [2.3, under the standing assumption (A) the number $\lambda=\mu_{*}$ is not an eigenvalue of $T_{2}(p, r)$. The proof of (3.7) uses this fact and is analogous to the proof of the corresponding lemma in 14. The coincidence of the spectra $\sigma\left(\mathscr{D}_{1}(P)\right)$ and $\sigma\left(T_{1}(p, r)\right)$ is proved along the same lines using the fact that the boundary condition $y^{[1]}(1)=0$ is equivalent to $u_{1}(1)=0$ in view of the relation $(v-r)(1)=0$. The last statement of the lemma is obtained by direct verification.

\section{TRANSFORMATION OPERATOR}

Lemma 3.2 suggests that we can try to use the spectra $\boldsymbol{\mu}$ and $\boldsymbol{\lambda}$ of the operator pencils $T_{1}(p, r)$ and $T_{2}(p, r)$ in order to find the related Dirac operators $\mathscr{D}_{j}(P), j=1,2$. Having determined the potential $P=$ $\left(p_{i j}\right)_{i, j=1}^{2}$ of $\ell(P)$, we then identify the potentials $p$ and $r$ of the operator pencil $T_{j}(p, r)$ as $p:=\left(p_{22}+p_{11}\right) / 2$ and $r:=-p_{12}-\int_{x}^{1}\left(p_{12}^{2}-p_{11} p_{22}\right)$.

However, the classical inverse spectral theory reconstructs a Dirac operator with potential in the AKNS form or in other canonical form. Thus to find the Dirac operators directly associated with the operator pencils $T_{j}(p, r)$, i.e. with potentials of the form (3.4), we have to transform the obtained Dirac operators in canonical form keeping the 
spectral data unchanged. This is done by means of the so-called transformation operators. In [14] such operators were constructed and some of their properties were discussed; see also [5, 28. In this section we shall focus our attention on some further properties of the transformation operators that are important for our reconstructing procedure.

Assume that $P$ and $Q$ are $2 \times 2$ matrix-valued potentials in $L_{2}\left((0,1), \mathcal{M}_{2}\right)$ and set

$$
\mathcal{D}_{0}:=\left\{\left(u_{1}, u_{2}\right)^{\mathrm{t}} \in W_{2}^{1}(0,1) \times W_{2}^{1}(0,1) \mid u_{2}(0)=0\right\} .
$$

The transformation operator $\mathscr{X}=\mathscr{X}(P, Q)$ between the Dirac operators $\ell(P)$ and $\ell(Q)$ on the set $\mathcal{D}_{0}$, i.e. the nontrivial operator satisfying the relation $\mathscr{X} \ell(P) \mathbf{u}=\ell(Q) \mathscr{X} \mathbf{u}$ for all $\mathbf{u} \in \mathcal{D}_{0}$, was constructed in [14] in the form

$$
\mathscr{X} \mathbf{u}(x)=R(x) \mathbf{u}(x)+\int_{0}^{x} K(x, s) \mathbf{u}(s) d s,
$$

where $R$ and $K$ are $2 \times 2$ matrix-valued functions of one and two variables respectively. Under the normalization $R(0)=I$, the operator $R$ is explicitly given [5] as

$$
R(x)=e^{\theta_{1}(x)}\left(\begin{array}{cc}
\cos \theta_{2}(x) & \sin \theta_{2}(x) \\
-\sin \theta_{2}(x) & \cos \theta_{2}(x)
\end{array}\right)=e^{\theta_{1}(x) I+\theta_{2}(x) J},
$$

with

$$
\begin{array}{r}
\theta_{1}(x)=\frac{1}{2} \int_{0}^{x} \operatorname{tr}[J(Q(s)-P(s))] d s, \\
\theta_{2}(x)=\frac{1}{2} \int_{0}^{x} \operatorname{tr}(Q(s)-P(s)) d s .
\end{array}
$$

Existence of the transformation operator $\mathscr{X}(P, Q)$ is guaranteed by the following two theorems.

Theorem 4.1 (14]). Assume that $P$ and $Q$ are in $L_{2}\left((0,1), \mathcal{M}_{2}\right)$. Then an operator $\mathscr{X}(P, Q)$ of the form (4.1), with $R$ obeying the condition $R(0)=I$ and a summable kernel $K$, is a transformation operator for $\ell(P)$ and $\ell(Q)$ on the set $\mathcal{D}_{0}$ if and only if the matrix-valued function $R$ is given by (4.2)-(4.3) and the kernel $K$ is a mild solution of the partial differential equation

$$
J \partial_{x} K(x, y)+\partial_{y} K(x, y) J=K(x, y) P(y)-Q(x) K(x, y)
$$

in the domain $\Omega:=\{(x, y) \mid 0<y<x<1\}$ satisfying for $0 \leq x \leq 1$ the boundary conditions

$$
\begin{aligned}
K(x, x) J-J K(x, x) & =J R^{\prime}(x)+Q(x) R(x)-R(x) P(x), \\
K_{12}(x, 0) & =K_{22}(x, 0)=0 .
\end{aligned}
$$


Theorem 4.2 (14]). Assume that matrix-valued functions $P$ and $Q$ are in $L_{2}\left((0,1), \mathcal{M}_{2}\right)$. Then the system (4.4) -(4.6) has a unique solution in the sense of distributions; moreover, this solution belongs to $L_{2}\left(\Omega, \mathcal{M}_{2}\right)$.

Throughout the rest of the paper, we shall assume that the matrixvalued potentials $P$ and $Q$ are Hermitian, i.e. that $P^{*}(x)=P(x)$ and $Q^{*}(x)=Q(x)$ a.e. on $[0,1]$. Then the corresponding Dirac operators $\mathscr{D}_{j}(P)$ and $\mathscr{D}_{j}(Q), j=1,2$, are self-adjoint and have simple discrete spectra. We denote by $\boldsymbol{\mu}(P)$ (resp. by $\boldsymbol{\mu}(Q)$ ) the spectrum of $\mathscr{D}_{1}(P)$ (resp. of $\left.\mathscr{D}_{1}(Q)\right)$ and by $\boldsymbol{\lambda}(P)$ (resp. by $\boldsymbol{\lambda}(Q)$ ) the spectrum of $\mathscr{D}_{2}(P)$ (resp. of $\mathscr{D}_{2}(Q)$ ). The sets $\boldsymbol{\mu}(P)$ and $\boldsymbol{\lambda}(P)$ interlace and their elements can be labelled by $n \in \mathbb{Z}$ so that $\mu_{n}=\pi\left(n-\frac{1}{2}\right)+\frac{1}{2} \int_{0}^{1} \operatorname{tr} P+$ $\mathrm{o}(1)$ and $\lambda_{n}=\pi n+\frac{1}{2} \int_{0}^{1} \operatorname{tr} P+\mathrm{o}(1)$ as $|n| \rightarrow \infty$ (see [28]); the spectra $\boldsymbol{\mu}(Q)$ and $\boldsymbol{\lambda}(Q)$ have similar properties.

Also, $\mathscr{X}=\mathscr{X}(P, Q)$ will stand for the transformation operator of the form (4.1) for the differential expressions $\ell(P)$ and $\ell(Q)$ on the domain $\mathcal{D}_{0}$, with $R$ given by (4.2) and (4.3). We shall write $\mathscr{X}=$ $\mathscr{R}+\mathscr{K}$, where $\mathscr{R} \mathbf{u}(x):=R(x) \mathbf{u}(x)$ is the operator of multiplication by $R$ and

$$
\mathscr{K} \mathbf{u}(x):=\int_{0}^{x} K(x, s) \mathbf{u}(s) d s
$$

is the corresponding integral operator. We observe that for Hermitian $P$ and $Q$ the functions $i \theta_{1}$ and $\theta_{2}$ are real valued; in particular, the operator $\mathscr{R}$ is unitary.

Remark 4.3. Let us note that if $u \in \mathcal{D}_{0}$, then the relation

$$
(\ell(P)-\lambda) \mathbf{u}=\mathbf{f}
$$

holds if and only if for $\mathbf{v}:=\mathscr{X} \mathbf{u}$ and $\mathbf{g}:=\mathscr{X} \mathbf{f}$ one gets

$$
(\ell(Q)-\lambda) \mathbf{v}=\mathbf{g} .
$$

Theorem 4.4. Assume that the matrix potentials $P$ and $Q$ are Hermitian. Then the spectra of the operators $\mathscr{D}_{j}(P)$ and $\mathscr{D}_{j}(Q), j=1,2$, coincide, i.e. $\boldsymbol{\mu}(P)=\boldsymbol{\mu}(Q)$ and $\boldsymbol{\lambda}(P)=\boldsymbol{\lambda}(Q)$, if and only if the transformation operator $\mathscr{X}(P, Q)$ for $\ell(P)$ and $\ell(Q)$ on the domain $\mathcal{D}_{0}$ only contains the unitary part $\mathscr{R}$ (i.e. $\mathscr{K}=0$ ) and $\theta_{2}(1)=\pi n$ for some $n \in \mathbb{Z}$.

Proof. Sufficiency. By assumption, the transformation operator $\mathscr{X}(P, Q)$ between $\ell(P)$ and $\ell(Q)$ only contains the multiplication operator $\mathscr{R}$. Denote by $\widetilde{\mathscr{D}}_{j}(P), j=1,2$, the operators related to $\mathscr{D}_{j}(Q)$ by

$$
\widetilde{\mathscr{D}}_{j}(P)=\mathscr{R}^{-1} \mathscr{D}_{j}(Q) \mathscr{R}, j=1,2 .
$$


Then $\widetilde{\mathscr{D}}_{j}(P)$ are unitarily equivalent to $\mathscr{D}_{j}(Q)$ and so $\sigma\left(\widetilde{\mathscr{D}}_{j}(P)\right)=$ $\sigma\left(\mathscr{D}_{j}(Q)\right)$. By Remark 4.3, $\widetilde{\mathscr{D}}_{j}(P)$ are Dirac operators acting via $\widetilde{\mathscr{D}}_{j}(P) \mathbf{u}=$ $\ell(P) \mathbf{u}$ on the domains which consist of those $\mathbf{u} \in L_{2}\left((0,1), \mathbb{C}^{2}\right)$ for which $\mathscr{R} \mathbf{u} \in \operatorname{dom} \mathscr{D}_{j}(Q)$. Recall that $R(0)=I$; also since $\theta_{2}(1)=\pi n$ for some $n \in \mathbb{Z}$, we have that $R(1)$ is a multiple of the identity matrix $I$. Therefore these $\mathbf{u}$ satisfy the same boundary conditions as $\mathscr{R} \mathbf{u}$. Thus $\operatorname{dom} \widetilde{\mathscr{D}}_{j}(P)=\operatorname{dom} \mathscr{D}_{j}(Q)=\operatorname{dom} \mathscr{D}_{j}(P)$. This means that $\mathscr{D}_{j}(P)=\widetilde{\mathscr{D}}_{j}(P)$, and so $\boldsymbol{\mu}(P)=\boldsymbol{\mu}(Q)$ and $\boldsymbol{\lambda}(P)=\boldsymbol{\lambda}(Q)$.

Necessity. Firstly note that as the spectra of $\mathscr{D}_{j}(P)$ and $\mathscr{D}_{j}(Q)$, $j=1,2$, coincide, the asymptotics of the eigenvalues also coincide thus yielding $\theta_{2}(1)=\pi n$ for some $n \in \mathbb{Z}$. To complete the proof it remains to show that $\mathscr{K}=0$.

Recall that the norming constant corresponding to an eigenvalue $\lambda$ of the operator $\mathscr{D}_{2}(P)$ is defined as $\|\mathbf{u}\|^{2}$, where $\mathbf{u}=\left(u_{1}, u_{2}\right)^{\mathrm{t}}$ is the eigenfunction of $\mathscr{D}_{2}(P)$ for $\lambda$ normalized by the initial conditions $u_{1}(0)=1$ and $u_{2}(0)=0$ [28. It follows from [3] that the norming constants for $\mathscr{D}_{2}(P)$ are uniquely determined by the spectra $\boldsymbol{\lambda}(P)$ and $\boldsymbol{\mu}(P)$ (although only AKNS potentials were treated in [3, the arguments therein are valid for all self-adjoint Dirac operators). Thus from the statement of the theorem we conclude that the sets of norming constants for $\mathscr{D}_{2}(P)$ and $\mathscr{D}_{2}(Q)$ coincide. By Lemma 4.4. of [14] this gives that $\mathscr{K}=0$.

Alternatively, to establish the necessity part of the above theorem, one can use arguments analogous to those in [5], where a similar statement but under different assumptions on $P$ and $Q$ was proved.

\section{ReConstruction of the PENCILS}

In this section, we study the problem of reconstructing the potentials $p$ and $r$ of the corresponding operator pencils $T_{j}(p, r), j=1,2$, from their spectra. Our aim is to prove Theorem 2.4, i.e. that given an arbitrary element $(\boldsymbol{\lambda}, \boldsymbol{\mu})$ of $S D$, there exist unique $p$ and $r$ such that $\boldsymbol{\mu}$ is the spectrum of the operator pencil $T_{1}(p, r)$ and $\boldsymbol{\lambda}$ is that of $T_{2}(p, r)$.

Fix therefore an arbitrary pair $(\boldsymbol{\lambda}, \boldsymbol{\mu})$ in SD. In what follows, $\lambda_{n}, n \in$ $\mathbb{Z}^{*}$, and $\mu_{n}, n \in \mathbb{Z}$, will stand respectively for elements of $\boldsymbol{\lambda}$ and $\boldsymbol{\mu}$. Note that the enumeration of $\mu_{n}$ and $\lambda_{n}$ fixes the shift $h$ in their asymptotics (2.1). We set $\mu_{*}$ to be the middle point of the interval $\left(\mu_{0}, \mu_{1}\right)$, i.e. $\mu_{*}:=\left(\mu_{0}+\mu_{1}\right) / 2$, and augment the sequence $\left(\lambda_{n}\right)_{n \in \mathbb{Z}^{*}}$ with an element $\lambda_{0}=\mu_{*}$; denote obtained sequence by $\boldsymbol{\lambda}^{*}$.

Recall now some facts from the inverse spectral theory for Dirac operators, which we shall use in our procedure, see [3, 7, 28]. Consider 
the set $\mathcal{Q}_{0}$ of $2 \times 2$ matrix-valued functions of the AKNS normal form, namely

$$
\mathcal{Q}_{0}:=\left\{Q_{0}=\left(\begin{array}{cc}
q_{1} & q_{2} \\
q_{2} & -q_{1}
\end{array}\right) \mid q_{j} \in L_{2, \mathbb{R}}(0,1)\right\} .
$$

It is known that the operators $\mathscr{D}_{1}\left(Q_{0}\right)$ and $\mathscr{D}_{2}\left(Q_{0}\right)$ with potential $Q_{0}$ from $\mathcal{Q}_{0}$ are self-adjoint and have simple discrete spectra. Their eigenvalues can be enumerated in increasing order as $\lambda_{n}\left(Q_{0}\right)$ and $\mu_{n}\left(Q_{0}\right)$, $n \in \mathbb{Z}$, respectively so that they satisfy the interlacing condition

$$
\mu_{n}\left(Q_{0}\right)<\lambda_{n}\left(Q_{0}\right)<\mu_{n+1}\left(Q_{0}\right)
$$

and obey the asymptotics

$$
\begin{aligned}
& \lambda_{n}\left(Q_{0}\right)=\pi n+\tilde{\lambda}_{n}\left(Q_{0}\right), \\
& \mu_{n}\left(Q_{0}\right)=\pi\left(n-\frac{1}{2}\right)+\tilde{\mu}_{n}\left(Q_{0}\right)
\end{aligned}
$$

with $\ell_{2}(\mathbb{Z})$-sequences $\left(\tilde{\lambda}_{n}\left(Q_{0}\right)\right)$ and $\left(\tilde{\mu}_{n}\left(Q_{0}\right)\right)$.

It is also known (see [3]) that for two sequences $\left(\mu_{n}\right)$ and $\left(\lambda_{n}\right)$ having the above properties there exists a unique potential $Q_{0}$ from $\mathcal{Q}_{0}$ such that the spectrum of $\mathscr{D}_{1}\left(Q_{0}\right)$ is $\left(\mu_{n}\right)$ and that of $\mathscr{D}_{2}\left(Q_{0}\right)$ is $\left(\lambda_{n}\right)$.

The above sequences $\boldsymbol{\mu}$ and $\boldsymbol{\lambda}^{*}$ differ from the spectra for Dirac operators with potentials of normal AKNS form only by the shift $h$ in their asymptotics, see (2.1). For $h \in \mathbb{R}$, we denote by

$$
\mathcal{Q}_{h}:=\left\{Q_{0}+h I \mid Q_{0} \in \mathcal{Q}_{0}\right\}
$$

the set of $h$-shifted AKNS matrix potentials; then the following result holds true.

Proposition 5.1. For an arbitrary pair $(\boldsymbol{\lambda}, \boldsymbol{\mu})$ from $S D$ take $\mu_{*}:=$ $\left(\mu_{0}+\mu_{1}\right) / 2$ and denote by $\boldsymbol{\lambda}^{*}$ the augmentation of $\boldsymbol{\lambda}$ with $\lambda_{0}=\mu_{*}$. Then there exists a unique potential $Q \in \mathcal{Q}_{h}$ such that $\boldsymbol{\mu}$ is the spectrum of the operator $\mathscr{D}_{1}(Q)$ and $\boldsymbol{\lambda}^{*}$ is that of $\mathscr{D}_{2}(Q)$.

For an arbitrary $P \in L_{2}\left((0,1), \mathcal{M}_{2}\right)$, we introduce the set

$$
\operatorname{Iso}(P):=\left\{\tilde{P} \in L_{2}\left((0,1), \mathcal{M}_{2}\right) \mid \boldsymbol{\mu}(\tilde{P})=\boldsymbol{\mu}(P), \boldsymbol{\lambda}(\tilde{P})=\boldsymbol{\lambda}(P)\right\}
$$

of isospectral potentials and for $\mu \in \mathbb{R}$ denote by $\mathcal{P}_{\mu}$ the set of all potentials of the form (3.4), i.e.

$$
\mathcal{P}_{\mu}:=\left\{P=\left(p_{i j}\right)_{i, j=1}^{2} \mid p_{i j} \in L_{2, \mathbb{R}}(0,1), p_{11}=\mu, p_{12}=p_{21}\right\} .
$$

Now we are going to prove that for every $\mu \in \mathbb{R}$ and $Q \in \mathcal{Q}_{h}$ there exists a unique potential $P$ from $\operatorname{Iso}(Q) \cap \mathcal{P}_{\mu}$. We start with the following lemma. 
Lemma 5.2. Suppose that $Q \in \mathcal{Q}_{h}$, i.e.

$$
Q=\left(\begin{array}{cc}
q_{1}+h & q_{2} \\
q_{2} & -q_{1}+h
\end{array}\right) .
$$

Then for every fixed $\mu \in \mathbb{R}$ there exists a unique potential $P \in \mathcal{P}_{\mu}$ such that, with $\mathcal{R}$ being the operator of multiplication by the matrix-valued function $R$ of (4.2)-(4.3), one has

$$
\mathscr{R} \ell(P)=\ell(Q) \mathscr{R} \text {. }
$$

Proof. First we note that the matrix $R$ given by (4.2) commutes with $J$, whence

$$
R^{-1}\left(J \frac{d}{d x}+Q\right) R=J \frac{d}{d x}+R^{-1} J R^{\prime}+R^{-1} Q R .
$$

Thus the relation (5.2) requires that

$$
\begin{aligned}
P & =R^{-1} J R^{\prime}+R^{-1} Q R \\
& =\left(h-\theta_{2}^{\prime}\right) I+\theta_{1}^{\prime} J+\left(\begin{array}{cc}
q_{1} \cos 2 \theta_{2}-q_{2} \sin 2 \theta_{2} & q_{1} \sin 2 \theta_{2}+q_{2} \cos 2 \theta_{2} \\
q_{1} \sin 2 \theta_{2}+q_{2} \cos 2 \theta_{2} & -q_{1} \cos 2 \theta_{2}+q_{2} \sin 2 \theta_{2}
\end{array}\right) .
\end{aligned}
$$

Now we fix an arbitrary $\mu \in \mathbb{R}$ and observe that the potential $P$ of (5.3) belongs to $\mathcal{P}_{\mu}$ if and only if $\theta_{1}(x)$ is identically zero and the following equality holds:

$$
-\theta_{2}^{\prime}+q_{1} \cos 2 \theta_{2}-q_{2} \sin 2 \theta_{2}+h=\mu .
$$

Clearly, there exists a unique solution of the above equation satisfying the initial condition

$$
\theta_{2}(0)=0 \text {. }
$$

This solution and $\theta_{1} \equiv 0$ verify the relation (4.3) with the given $Q$ and with $P$ of (5.3). The potential $P$ is explicitly given by (5.3) with $\theta_{1} \equiv 0$ and $\theta_{2}$ as above and by construction it belongs to $\mathcal{P}_{\mu}$ and satisfies (5.2). The proof is complete.

The next result states existence and uniqueness of the potential $P$ from $\mathcal{P}_{\mu}$ belonging to $\operatorname{Iso}(Q)$.

Theorem 5.3. Let $\mu, h \in \mathbb{R}$ and assume that $Q \in \mathcal{Q}_{h}$ is such that $\lambda=\mu$ is an eigenvalue of the Dirac operator $\mathscr{D}_{2}(Q)$. Then there exists a unique $P \in \mathcal{P}_{\mu}$ belonging to $\operatorname{Iso}(Q)$, i.e. $\operatorname{Iso}(Q) \cap \mathcal{P}_{\mu}=\{P\}$.

Proof. Observe first that if $P \in \operatorname{Iso}(Q)$, then by Theorem 4.4 the transformation operator between $\ell(P)$ and $\ell(Q)$ on $\mathcal{D}_{0}$ is just the operator $\mathscr{R}$ of multiplication by the matrix-valued function $R$ given by (4.2)-(4.3). If, moreover, $P$ should belong to $\mathcal{P}_{\mu}$, then by Lemma $5.2 P$ should be 
given by (15.3) with $\theta_{1} \equiv 0$ and $\theta_{2}$ solving (5.4)-(5.5). It remains to prove that the potential given by (5.3) - (5.5) indeed belongs to $\operatorname{Iso}(Q)$. By Theorem 4.4, to this end it suffices to show that $\theta_{2}(1)=\pi n$ for some $n \in \mathbb{Z}$.

Consider the operator $\widetilde{\mathscr{D}}_{2}(P):=\mathscr{R}^{-1} \mathscr{D}_{2}(Q) \mathscr{R}$. Clearly, $\widetilde{\mathscr{D}}_{2}(P)$ is a Dirac operator acting via $\widetilde{\mathscr{D}}_{2}(P) \mathbf{u}=\ell(P) \mathbf{u}$ on the domain consisting of those $\mathbf{u}$ for which $\mathcal{R} \mathbf{u}$ belongs to $\operatorname{dom} \mathscr{D}_{2}(Q)$. By construction, $\sigma\left(\widetilde{\mathscr{D}}_{2}(P)\right)=\sigma\left(\mathscr{D}_{2}(Q)\right)$ and thus $\lambda=\mu$ is in the spectrum of $\widetilde{\mathscr{D}}_{2}(P)$. Denote by $\mathbf{u}_{0}=\left(u_{1}, u_{2}\right)^{\mathrm{t}}$ the eigenfunction of $\widetilde{\mathscr{D}}_{2}(P)$ corresponding to $\lambda=\mu$. Then $\mathbf{v}_{0}=\left(v_{1}, v_{2}\right)^{\mathrm{t}}:=\mathscr{R} \mathbf{u}_{0}$ is an eigenfunction of $\mathscr{D}_{2}(Q)$ corresponding to $\lambda$, so that $v_{2}(0)=v_{2}(1)=0$. The equality $\ell(P) \mathbf{u}_{0}=\mu \mathbf{u}_{0}$ yields the relation $u_{2}^{\prime}-v u_{2}=0$. Since $R(0)=I$, we have $u_{2}(0)=v_{2}(0)=0$, which together with the above relation gives that $u_{2}$ is identically zero. Therefore

$$
\mathbf{v}_{0}(1)=R(1) \mathbf{u}_{0}(1)=\left(\begin{array}{c}
u_{1}(1) \cos \theta_{2}(1) \\
-u_{1}(1) \sin \theta_{2}(1)
\end{array}\right)
$$

But $v_{2}(1)=0$ and $u_{1}(1) \neq 0$ giving that $\theta_{2}(1)=\pi n, n \in \mathbb{Z}$.

With these preliminaries at hand we can prove the main results of the paper.

Proof of Theorem 2.4. Existence. Given a pair $(\boldsymbol{\lambda}, \boldsymbol{\mu})$ from $S D$, we set $\mu_{*}:=\left(\mu_{0}+\mu_{1}\right) / 2$ and augment $\boldsymbol{\lambda}$ with $\lambda_{0}:=\mu_{*}$ to obtain $\boldsymbol{\lambda}^{*}$. Using Proposition 5.1, we find the potential $Q$ in $\mathcal{Q}_{h}$ such that $\boldsymbol{\mu}$ is the spectrum of the operator $\mathscr{D}_{1}(Q)$ and $\boldsymbol{\lambda}^{*}$ is that of $\mathscr{D}_{2}(Q)$. Theorem 5.3 gives that for this $Q$ we can find $P \in \mathcal{P}_{\mu_{*}}$ belonging to Iso $(Q)$, i.e. such that $\boldsymbol{\mu}$ and $\boldsymbol{\lambda}^{*}$ are respectively the spectra of $\mathscr{D}_{1}(P)$ and $\mathscr{D}_{2}(P)$. In view of Lemma 3.2 this means that $\boldsymbol{\mu}$ and $\boldsymbol{\lambda}$ are the spectra of $T_{1}(p, r)$ and $T_{2}(p, r)$ with

$$
p=\frac{p_{22}+p_{11}}{2}, \quad r:=-p_{12}-\int_{x}^{1}\left(p_{12}^{2}-p_{11} p_{22}\right) .
$$

The almost interlacing of $\boldsymbol{\lambda}$ and $\boldsymbol{\mu}$ gives that $T_{1}(p, r)$ is hyperbolic (see [38]), i.e. it satisfies assumption (A).

This completes the proof of existence.

Uniqueness. Suppose there are two pairs of potentials $p, r$ and $\hat{p}, \hat{r}$ such that $\sigma\left(T_{1}(p, r)\right)=\sigma\left(T_{1}(\hat{p}, \hat{r})\right)=: \boldsymbol{\mu}$ and $\sigma\left(T_{2}(p, r)\right)=\sigma\left(T_{2}(\hat{p}, \hat{r})\right)=$ : $\lambda$. Then (see [38] ) for every $\mu \in\left(\mu_{0}, \mu_{1}\right)$ the operators $T_{1}(p, r)(\mu)$ and $T_{1}(\hat{p}, \hat{r})(\mu)$ are negative, i.e. the assumption (A) holds for operator pencils $T_{1}(p, r)$ and $T_{1}(\hat{p}, \hat{r})$.

Set $\mu_{*}:=\left(\mu_{0}+\mu_{1}\right) / 2$; then the pencils $T_{j}(p, r)$ and $T_{j}(\hat{p}, \hat{r}), j=$ 1,2 , lead to two Dirac operators with potentials $P$ and $\hat{P}$ in $\mathcal{P}_{\mu_{*}}$ as 
explained in Section 3 and to two Dirac operators with potentials $Q$ and $\hat{Q}$ in $\mathcal{Q}_{h}$, with the same $h$ as explained at the beginning of this section. By construction, $\boldsymbol{\mu}(Q)=\boldsymbol{\mu}(\hat{Q})=\boldsymbol{\mu}$ and $\boldsymbol{\lambda}(Q)=\boldsymbol{\lambda}(\hat{Q})=$ $\boldsymbol{\lambda} \cup\left\{\mu_{*}\right\}$, whence $Q=\hat{Q}$ by Proposition 5.1. By Lemma 3.2 we have that $\boldsymbol{\mu}(P)=\boldsymbol{\mu}(\hat{P})$ and $\boldsymbol{\lambda}(P)=\boldsymbol{\lambda}(\hat{P})$ and thus $P$ and $\hat{P}$ belong to the same isospectral set $\operatorname{Iso}(Q)$. In view of Theorem 5.3 this yields that $P=\hat{P}$. Therefore, $\hat{p}=\hat{p}$ and $\hat{r}=\hat{r}$, which completes the proof.

\section{ReCOnstructing Algorithm}

To summarize let us formulate the algorithm of reconstruction of the operator pencils $T_{1}(p, r)$ and $T_{2}(p, r)$.

Suppose we have a pair of sequences $(\boldsymbol{\lambda}, \boldsymbol{\mu})$ from $S D$. Then the reconstructing procedure consists of the following steps:

(1) Take $\mu_{*}:=\left(\mu_{1}+\mu_{2}\right) / 2$ and augment the given sequence $\boldsymbol{\lambda}$ with an element $\lambda_{0}=\mu_{*}$;

(2) Use the obtained spectral data to construct the Dirac operators $\mathcal{D}_{1}(Q)$ and $\mathcal{D}_{2}(Q)$ with the potential $Q$ of the $h$-shifted AKNS form;

(3) Find the corresponding potential $P \in \mathcal{P}_{\mu_{*}} \cap \operatorname{Iso}(Q)$ using (5.3) and (5.4);

(4) Compute the potentials $p$ and $r$ using formulae (5.6).

We conclude this paper with some remarks. Firstly, note that the described approach can be used to reconstruct energy-dependent SturmLiouville equations subject to other boundary conditions or under different smoothness assumptions on the potentials, e.g. in the case when $p$ and $r$ are from $L_{s}(0,1)$ with $s \geq 1$ or belong to $W_{2}^{s}(0,1)$ with $s \geq 0$, cf. [17, 43].

Secondly, the considered method is also applicable to the inverse problems of reconstructing the quadratic pencil from different sets of spectral data, for example, the spectrum and the norming constants [14] or Hochstadt-Lieberman mixed data (cf. [12]). Finally, one can establish an analogue of the Hochstadt-type perturbation formulas when finitely many eigenvalues in one spectrum have been changed (cf. [11]).

\section{REFERENCES}

[1] T. Aktosun and C. van der Mee. Scattering and inverse scattering for the 1-D Schrödinger equation with energy-dependent potentials. J. Math. Phys., 32(10):2786-2801, 1991. 
[2] S. Albeverio, F. Gesztesy, R. Høegh-Krohn, and H. Holden. Solvable Models in Quantum Mechanics. With an appendix by Pavel Exner. 2nd revised ed. Providence, RI: AMS Chelsea Publishing, 2005.

[3] S. Albeverio, R. Hryniv, and Y. Mykytyuk. Inverse spectral problems for Dirac operators with summable potentials. Russ. J. Math. Phys., 12(4):406-423, 2005.

[4] S. Albeverio and P. Kurasov. Singular Perturbations of Differential Operators. Solvable Schrödinger Type Operators. Cambridge: Cambridge University Press, 1999.

[5] S. Cox and R. Knobel. An inverse spectral problem for a nonnormal first order differential operator. Integral Equations Operator Theory, 25(2):147-162, 1996.

[6] K.-J. Engel and R. Nagel. One-parameter Semigroups for Linear Evolution Equations, volume 194 of Graduate Texts in Mathematics. Springer-Verlag, New York, 2000.

[7] M. G. Gasymov and T. T. Džabiev. Solution of the inverse problem by two spectra for the Dirac equation on a finite interval. Akad. Nauk Azerbaŭdžan. SSR Dokl., 22(7):3-6, 1966.

[8] M. G. Gasymov and G. S. Gusernov. Determination of a diffusion operator from spectral data. Akad. Nauk Azerbaùdzhan. SSR Dokl., 37(2):19-23, 1981.

[9] G. S. Guseĭnov. Inverse spectral problems for a quadratic pencil of SturmLiouville operators on a finite interval. In Spectral theory of operators and its applications, No. 7 (Russian), pages 51-101. "Èlm", Baku, 1986.

[10] I. M. Guselnov and I. M. Nabiev. An inverse spectral problem for pencils of differential operators. Mat. Sb., 198(11):47-66, 2007.

[11] H. Hochstadt. The inverse Sturm-Liouville problem. Comm. Pure Appl. Math., 26:715-729, 1973.

[12] H. Hochstadt and B. Lieberman. An inverse Sturm-Liouville problem with mixed given data. SIAM J. Appl. Math., 34(4):676-680, 1978.

[13] R. Hryniv and N. Pronska. Oscillation results for energy-dependent SturmLiouville equation. In preparation.

[14] R. Hryniv and N. Pronska. Inverse spectral problem for energy-dependent Sturm-Liouville equation. ArXiv:1203.4851v1, 2012.

[15] R. O. Hryniv and Y. V. Mykytyuk. 1-D Schrödinger operators with periodic singular potentials. Methods Funct. Anal. Topology, 7(4):31-42, 2001.

[16] R. O. Hryniv and Y. V. Mykytyuk. Inverse spectral problems for SturmLiouville operators with singular potentials. II. Reconstruction by two spectra. In Functional analysis and its applications, volume 197 of North-Holland Math. Stud., pages 97-114. Elsevier Sci. B. V., Amsterdam, 2004.

[17] R. O. Hryniv and Y. V. Mykytyuk. Inverse spectral problems for SturmLiouville operators with singular potentials. IV. Potentials in the Sobolev space scale. Proc. Edinb. Math. Soc. (2), 49(2):309-329, 2006.

[18] M. Jaulent. On an inverse scattering problem with an energy-dependent potential. Ann. Inst. H. Poincaré Sect. A (N.S.), 17:363-378, 1972.

[19] M. Jaulent and C. Jean. The inverse $s$-wave scattering problem for a class of potentials depending on energy. Comm. Math. Phys., 28:177-220, 1972.

[20] M. Jaulent and C. Jean. The inverse problem for the one-dimensional Schrödinger equation with an energy-dependent potential. I. Ann. Inst. H. Poincaré Sect. A (N.S.), 25(2):105-118, 1976. 
[21] M. Jaulent and C. Jean. The inverse problem for the one-dimensional Schrödinger equation with an energy-dependent potential. II. Ann. Inst. H. Poincaré Sect. A (N.S.), 25(2):119-137, 1976.

[22] P. Jonas. On the spectral theory of operators associated with perturbed KleinGordon and wave type equations. J. Oper. Theory, 29(2):207-224, 1993.

[23] Y. Kamimura. Energy dependent inverse scattering on the line. Differential Integral Equations, 21(11-12):1083-1112, 2008.

[24] T. Kappeler, P. Perry, M. Shubin, and P. Topalov. The Miura map on the line. Int. Math. Res. Not., (50):3091-3133, 2005.

[25] T. Kato. Perturbation Theory for Linear Operators. Die Grundlehren der mathematischen Wissenschaften, Band 132. Springer-Verlag New York, Inc., New York, 1966.

[26] H. Langer, B. Najman, and C. Tretter. Spectral theory of the Klein-Gordon equation in Pontryagin spaces. Comm. Math. Phys., 267:156-180, 2006.

[27] H. Langer, B. Najman, and C. Tretter. Spectral theory of the Klein-Gordon equation in Krein spaces. Proc. Edinb. Math. Soc., II. Ser., 51(3):711-750, 2008.

[28] B. M. Levitan and I. S. Sargsjan. Sturm-Liouville and Dirac Operators, volume 59 of Mathematics and its Applications (Soviet Series). Kluwer Academic Publishers Group, Dordrecht, 1991. Translated from the Russian edition (Nauka Publ., Moscow, 1988).

[29] F. G. Maksudov and G. S. Guselnov. On the solution of the inverse scattering problem for a quadratic pencil of one-dimensional Schrödinger operators on the whole axis. Dokl. Akad. Nauk SSSR, 289(1):42-46, 1986.

[30] F. G. Maksudov and G. S. Guseĭnov. An inverse scattering problem for a quadratic pencil of Sturm-Liouville operators on the full line. In Spectral theory of operators and its applications, No. 9 (Russian), pages 176-211. "Èlm", Baku, 1989.

[31] A. S. Markus. Introduction to the Spectral Theory of Polynomial Operator Pencils, volume 71 of Translations of Mathematical Monographs. American Mathematical Society, Providence, RI, 1988. Translated from the Russian edition ("Shtiintsa", Kishinev, 1986). With an appendix by M. V. Keldysh.

[32] A. A. Nabiev. Inverse scattering problem for the Schrödinger-type equation with a polynomial energy-dependent potential. Inverse Problems, 22(6):20552068, 2006.

[33] A. A. Nabiev and I. M. Guseinov. On the Jost solutions of the Schrödinger-type equations with a polynomial energy-dependent potential. Inverse Problems, 22(1):55-67, 2006.

[34] I. M. Nabiev. The inverse spectral problem for the diffusion operator on an interval. Mat. Fiz. Anal. Geom., 11(3):302-313, 2004.

[35] I. M. Nabiev. An inverse quasiperiodic problem for a diffusion operator. Dokl. Akad. Nauk, 415(2):168-170, 2007.

[36] B. Najman. Eigenvalues of the Klein-Gordon equation. Proc. Edinb. Math. Soc. (2), 26:181-190, 1983.

[37] N. Pronska. Asymptotics of eigenvalues and eigenfunctions of energydependent Sturm-Liouville operators. In preparation.

[38] N. Pronska. Spectral properties of Sturm-Liouville operators with singular energy-dependent potentials. In preparation. 
[39] D. H. Sattinger and J. Szmigielski. Energy dependent scattering theory. Differential Integral Equations, 8(5):945-959, 1995.

[40] A. M. Savchuk and A. A. Shkalikov. Sturm-Liouville operators with singular potentials. Mat. Zametki, 66(6):897-912, 1999.

[41] A. M. Savchuk and A. A. Shkalikov. Sturm-Liouville operators with distribution potentials. Tr. Mosk. Mat. Obs., 64:159-212, 2003.

[42] A. M. Savchuk and A. A. Shkalikov. Inverse problem for Sturm-Liouville operators with distribution potentials: reconstruction from two spectra. Russ. J. Math. Phys., 12(4):507-514, 2005.

[43] A. M. Savchuk and A. A. Shkalikov. On the eigenvalues of the Sturm-Liouville operator with potentials in Sobolev spaces. Mat. Zametki, 80(6):864-884, 2006.

[44] M. Tsutsumi. On the inverse scattering problem for the one-dimensional Schrödinger equation with an energy dependent potential. J. Math. Anal. Appl., 83(1):316-350, 1981.

[45] C. van der Mee and V. Pivovarchik. Inverse scattering for a Schrödinger equation with energy dependent potential. J. Math. Phys., 42(1):158-181, 2001.

[46] M. Yamamoto. Inverse eigenvalue problem for a vibration of a string with viscous drag. J. Math. Anal. Appl., 152:20-34, 1990.

[47] C.-F. Yang and Y.-X. Guo. Determination of a differential pencil from interior spectral data. J. Math. Anal. Appl., 375(1):284-293, 2011.

(N.P.) Institute for Applied Problems of Mechanics and MathematICS, 3B NaUkova St., 79601 Lviv, Ukraine

E-mail address: nataliya.pronska@gmail.com 